\title{
GDF15(MIC1) H6D Polymorphism Does Not Influence Cardiovascular Disease in a Latin American Population with Rheumatoid Arthritis
}

\author{
Jenny Amaya-Amaya, ${ }^{1}$ Adriana Rojas-Villarraga, ${ }^{1}$ Nicolas Molano-Gonzalez, ${ }^{1}$ \\ Laura Montoya-Sánchez, ${ }^{1}$ Swapan K. Nath, ${ }^{2}$ and Juan-Manuel Anaya ${ }^{1}$ \\ ${ }^{1}$ Center for Autoimmune Diseases Research (CREA), School of Medicine and Health Sciences, Universidad del Rosario, \\ Carrera 24 No. 63C-69, Bogotá, Colombia \\ ${ }^{2}$ Arthritis and Clinical Immunology Research Program, Oklahoma Medical Research Foundation, 825 NE 13th Street, \\ Oklahoma City, OK 73104, USA
}

Correspondence should be addressed to Juan-Manuel Anaya; anayajm@gmail.com

Received 20 July 2014; Accepted 3 February 2015

Academic Editor: Mario Clerici

Copyright (C) 2015 Jenny Amaya-Amaya et al. This is an open access article distributed under the Creative Commons Attribution License, which permits unrestricted use, distribution, and reproduction in any medium, provided the original work is properly cited.

\begin{abstract}
Objective. Rheumatoid arthritis (RA) is the most common autoimmune arthropathy worldwide. The increased prevalence of cardiovascular disease (CVD) in RA is not fully explained by classic risk factors. The aim of this study was to determine the influence of rs1058587 SNP within GDF15(MIC1) gene on the risk of CVD in a Colombian RA population. Methods. This was a cross-sectional analytical study in which 310 consecutive Colombian patients with RA and 228 age- and sex-matched controls were included and assessed for variables associated with CVD. The mixed cluster methodology based on multivariate descriptive methods such as principal components analysis and multiple correspondence analyses and regression tree (CART) predictive model were performed. Results. Of the 310 patients, $87.4 \%$ were women and CVD was reported in $69.5 \%$. Significant differences concerning GDF15 polymorphism were not observed between patients and controls. Mean arterial pressure, current smoking, and some clusters were significantly associated with CVD. Conclusion. GDF15 (rs1058587) does not influence the development of CVD in the population studied.
\end{abstract}

\section{Introduction}

Rheumatoid arthritis (RA) is the most common autoimmune arthropathy worldwide. It is a chronic, multifactorial, and systemic disease characterized by the presence of long-standing inflammation $[1,2]$. As with most autoimmune diseases, RA predominantly affects women $[2,3]$, and its prevalence had been reported to be between 0.3 and $1.6 \%$ [4]. In addition to diarthrodial joints, RA can damage virtually any organ thus leading to potential extra-articular manifestations (EAMs), including cardiovascular disease (CVD) $[5,6]$. CVD is the major predictor of poor prognosis and represents the main cause of death in this population $[7,8]$. It accounts for $30-$ $50 \%$ of all deaths in RA patients [9] and the prevalence of CVD in RA Latin American population has been estimated to be around $35 \%[8]$.
In the etiological and pathogenic mechanisms of CVD in RA the inflammatory activity plays an important role. Several studies assessing traditional risk factors for CVD in RA have been published [10]. Nevertheless, the increased prevalence of cardiovascular events in RA is not fully explained by these classic risk factors. Nontraditional risk factors have also been identified and categorized into three groups: genetic, RArelated, and others [7]. Recently, an association between a nonsynonymous single nucleotide polymorphism (SNP) at growth differentiation factor 15 (GDF15) gene (i.e., rs1058587) and CVD in RA patients was reported [11].

GDF15, also known as macrophage inhibitory cytokine-1 (MIC1), depending on the tissue that was described, is a growth and differentiation factor which belongs to the superfamily of transforming growth factor-beta (TGF- $\beta$ ). GDF15 
plays multiple roles in various pathologies such as CVD, obesity, inflammation, and cancer due to its anti-inflammatory, antiproliferative, and antitumoral properties [11-13].

Its mechanism of action is not fully elucidated but it is believed that MIC1 activates the TGF- $\beta$ receptors. Specifically in inflammation, it can limit the later phases of macrophage activation, inhibit the production of tumoral necrosis factor$\alpha$ (TNF- $\alpha$ ) induced by lipopolysaccharides, and also regulate the role of the proinflammatory cytokine interleukin6 (IL-6) [10]. Additionally, MIC1 influences metabolism of carbohydrates and lipids. For instance, patients with obesity and type 2 diabetes mellitus (T2DM) have the highest serum concentrations of this factor [14].

Previous studies have found that some GDF15(MIC1) polymorphisms are associated with different atherothrombotic manifestations such as stroke, transient ischemic attack, deep vein thrombosis, and pulmonary embolism. GDF15(MIC1) had also been associated with RA per se, proposing that it acts as a proinflammatory cytokine as well as a common etiologic factor for RA and CVD [11, 12]. The aim of this study was to determine the influence of rs1058587 SNP within GDF15(MIC1) gene on the risk of CVD in a Colombian RA population.

\section{Methods}

2.1. Study Population. This was a cross-sectional analytical study in which 310 consecutive Colombian patients with RA and 228 age- and sex-matched controls were included. The sample size was not calculated. This was a nonprobability sample. The subjects were seen at the Center for Autoimmune Diseases Research (CREA) in Medellín and Bogotá, Colombia. Patients fulfilled the 1987 American College of Rheumatology (ACR) classification criteria for RA [15]. This study was undertaken between February 2008 and April 2010 and done in compliance with Act $008430 / 1993$ by Ministry of Health of the Republic of Colombia. The institutional review board of the Universidad del Rosario approved the study design.

Each patient was evaluated by a rheumatologist. The information on patient sociodemographic and cumulative clinical and laboratory data was obtained by interview, physical examination, and chart review. Household description by questionnaire and clinical evaluation of the affected family members was done with the same methodology as above. All data were collected in an electronic and secure database.

Sociodemographic variables included age at RA onset, disease duration, educational status, socioeconomic status (SES), current occupation, smoking habits, and physical activity. Definitions of these variables are as follows. Age at onset (AOD) is the age at which patients began to suffer from pain, typical morning stiffness (more than 1 hour), and symmetrical inflammation of hand and/or foot joints. Disease duration is the difference between AOD and the date of first participation in the study. Educational level was recorded as the number of years of education and was also divided into two groups-more or less than 9 years of education based on the General Law of Education in Colombia laws [16, 17]. SES was categorized on the basis of national legislation and was divided into high status, medium status, and low status. Information on current occupational status was also requested. Familial autoimmunity (FA) was defined as the presence of any $\mathrm{AD}$ in first degree relatives (FDRs) of the proband [18].

Erosions were defined as having at least one unequivocal cortical bone defect evaluated by a rheumatologist [19]. EAMs were defined as the presence of at least one of the following: skin and lung nodules, skin ulcerations, episcleritis, vasculitis, neuropathy, pleural effusion, pulmonary hypertension, or embolism. CVD was categorized as positive if any of the following variables were present: hypertension, coronary artery disease (CAD), occlusive arterial disease, carotid disease, or thrombosis [20]. Regarding medical treatment, current or past use of methotrexate and other conventional disease modifying antirheumatic drugs (DMARDs) such as sulfasalazine, D-penicillamine, gold salts, and leflunomide; biological therapy (etanercept, infliximab, adalimumab, abatacept, tocilizumab, and rituximab); and azathioprine and cyclosporine were also assessed. In addition, steroid therapy (prednisolone, methylprednisolone, and deflazacort) and antimalarials (chloroquine and hydroxychloroquine) were taken into account.

Autoantibodies including rheumatoid factor (RF), anticitrullinated protein (ACPA), and anti-nuclear antibodies (ANA) were extracted from the patient's clinical record.

2.2. Assessment of Traditional Risk Factors for CVD. Patients were assessed for traditional CVD risk factors including current age ( $\geq 45$ and $\geq 55$ years for men and women, resp.) [21] and history of premature CAD in FDR [22], and all individuals were asked about smoking and physical activity [23]. T2DM was defined as having a fasting plasma glucose level $\geq 7 \mathrm{mmol} / \mathrm{L}(126 \mathrm{mg} / \mathrm{dL})$ or taking any antidiabetic agents at the time of assessment [24]. A diagnosis of dyslipidemia was given if the patient had (a) hypercholesterolemia, defined as taking lipid-lowering medication as a surrogate or having a fasting plasma total cholesterol $\geq 200 \mathrm{mg} /$ $\mathrm{dL}$, (b) $\mathrm{HDL} \leq 40 \mathrm{mg} / \mathrm{dL}$, (c) hypertriglyceridemia (triglycerides $\geq 150 \mathrm{mg} / \mathrm{dL}$ ), or (d) elevated LDL ( $\geq 100 \mathrm{mg} / \mathrm{dL}$ ) [25] and hypertension (defined as having a blood pressure $\geq 140 / 90 \mathrm{mmHg}$ or using any antihypertensive medication). Systolic and diastolic blood pressures were measured twice with at least a 15-minute interval in between and the averages were recorded [26].

2.3. Anthropometric Measurements. A body mass index (BMI) $\geq 25 \mathrm{~kg} / \mathrm{m}^{2}$ (overweight and obesity) was considered abnormal [27]. Values of waist circumference $(\geq 102 \mathrm{~cm}$ for men and $\geq 88 \mathrm{~cm}$ for women) and waist-to-hip ratio (WHR) $\geq 0.9$ for men and $\geq 0.85$ for women were considered indicators of abdominal obesity. Waist circumference was measured around the midpoint between the lowest rib and the iliac crest after exhaling and viewed from the front. Hip circumference was measured at the point of maximum extension of the buttocks when viewed from the side [28]. Abnormal WHR values are consistent with National Cholesterol Education Program Adult Treatment Panel III and World Health Organization 
definitions $[29,30]$. The above measurements were used to find the most reliable predictors of vascular involvement.

\subsection{CVD and Assessment of Carotid Intima-Media Thickness} (IMT). CVD was categorized as positive if any of the following subphenotypes were present: hypertension, coronary artery disease (CAD), occlusive arterial disease, carotid disease, or thrombosis [20]. In addition, an expert blind to the patients' clinical records performed ultrasound measurements of the IMT from the common carotid artery. Highresolution, 2-dimensional images were obtained using an ultrasound machine (Agilent-Hewlett-Packard, Santa Clara, CA). A $7.5 \mathrm{MHz}$ linear-array transducer with an axial resolution of $0.15 \mathrm{~mm}$ and a penetration depth from 1.0 to $5.0 \mathrm{~cm}$ was used [31]. The patient rested on the examination table for 15 minutes before the initial carotid ultrasound scan. The measurement was applied to the far wall of the right and left carotid arteries. Following R. Salonen and J. T. Salonen [32], a B-mode screening method was used with electronic calipers within $10 \mathrm{~mm}$ proximal to the common carotid bifurcation in a temperature-controlled room $\left(22^{\circ} \mathrm{C}\right.$ to $24^{\circ} \mathrm{C}$ ) [33]. Ultrasound images were recorded on videotape (Sony MD385). IMT was measured at the site of the greatest thickness and at 2 additional points: $1 \mathrm{~cm}$ upstream and $1 \mathrm{~cm}$ downstream from this site. The average of these 3 values was computed. Since carotid IMT greater than $0.90 \mathrm{~mm}$ is included among the definitions of subclinical organ damage [34], we established this point as having severe subclinical atherosclerosis (AT). The reproducibility of the IMT was evaluated in 12 volunteers, by taking 2 measurements one month apart; obtaining an intraclass correlation of 0.98 .

2.5. Genotyping. Genomic DNA was extracted from buffycoat cells using standard methods. All the identified genomic DNA samples were genotyped for GDF15(MIC1) rs1058587 at the Oklahoma Medical Research Foundation using the TaqMan allelic discrimination assay (Applied Biosystems). The GDF15(MIC1) gene, located at band p13.11 on chromosome 19, has two exons that encode the 308-amino acid GDF15(MIC1) polypeptide, consisting of a 29 -amino acid signal peptide, a 167-amino acid propeptide, and a 112-amino acid mature protein. Cleavage of the propeptide allows the mature protein to be secreted as a disulfide-linked homodimer. rs 1058587 SNP is at codon 202 (CAC to GAC) and results in a histidine to aspartic acid substitution at position 6 of the mature protein (H6D) (http://www.ncbi.nlm.nih.gov/gene/9518).

2.6. Statistical Analysis. Hardy-Weinberg equilibrium was checked in each genotypic marker. Univariate analyses were performed as follows: the categorical variables were analyzed by the frequencies. The quantitative continuous variables were expressed as the mean and standard deviation, as well as the median and range. Bivariate analysis of CVD outcome (subphenotypes and IMT) versus genetic, clinical, and demographic variables was assessed by means of chi square and Kruskal-Wallis test. The mixed cluster methodology proposed by Morineau et al. [35] based on multivariate descriptive methods such as principal components analysis
(PCA) and multiple correspondence analysis (MCA) was performed to resume some sets of variables that have strong associations. For example, AOD and duration of disease were analyzed in this setting to derive three groups that resemble the associations of these variables (i.e., time cluster). The same was done for SES and educational level deriving four groups (i.e., sod-cluster) and, for Sjögren's syndrome (SS), EAM, and comorbidity three groups were determined (i.e., clinical cluster). This allows the integration of these sets of variables that are confounded, via the new clusters, in association models like logistic regression and classification and regression trees (CART).

CART were used to find predictive factors for CVD in RA. The CART model was adjusted in the Salford Predictive Modeler software v7 using different splitting rules and crossvalidation samples to accurately estimate the relative errors of the classification tree. As the independent factors, the model included the variables that were statistically significant in bivariate analyses and those variables that were biologically plausible, as well as the new clusters derived. Bivariate and multivariate analyses were performed in R 3.0.2 [36].

\section{Results}

Of the 310 patients, $87.4 \%$ were women and CVD was reported in $69.5 \%$. The SNP rs 1058587 was in Hardy-Weinberg equilibrium $(P>0.05)$. Allelic frequencies corresponding to rs1058587 SNP C and G were $82.7 \%$ and $17.5 \%$, respectively. The most frequent genotype was CC (68.4\%). The frequencies of RF and ACPA were $68.3 . \%$ and $80.4 \%$, respectively. Characteristics of the cohort are illustrated in Table 1. Significant differences concerning GDF15 polymorphism (rs1058587) were not observed between patients and controls (Table 2).

Data analysed by PCA and MCA were resumed in clusters under clinical, time, and sociodemographic categories. The clinical clusters was gathered into 3 groups: (1) the highest frequency comorbidities (e.g., T2DM, dyslipidemia, osteoporosis, acid peptic disease, kidney disease, depression, periodontal disease, and fibromyalgia), not having polyautoimmunity with SS and moderate frequency of EAM; (2) presence of comorbidities and polyautoimmunity with SS and the highest frequency of EAM; (3) absence of comorbidities, not having polyautoimmunity with SS and lower frequency of EAM. The time clusters was categorized into 3 groups: (1) later AOD and shorter duration of the disease; (2) earlier AOD and longer duration of the disease; (3) earlier AOD and shorter duration of the disease. Finally, the sod-clusters was classified into 4 groups: (1) medium SES and the highest educational level; (2) medium SES and lower educational level; (3) the highest SES and higher educational level; and (4) the lowest SES and higher educational level.

The patients with CVD were significantly older and had a later AOD of RA. Duration of EAM, mean arterial pressure, current smoking, clinical cluster number 1 , and time cluster number 1 were all risk factors significantly associated with CVD. Associations between GDF15 rs1058587 SNP and CVD 
TABLE 1: Demographic and clinical characteristics of 310 patients with RA.

\begin{tabular}{|c|c|}
\hline Variable & Median (range) \\
\hline Age (y) & $37(20-54)$ \\
\hline Age at onset $(y)$ & $50(26-67)$ \\
\hline Duration of the disease (y) & $12(1-20)$ \\
\hline BMI & $29(19-37)$ \\
\hline EAM duration $(\mathrm{m}$; media $\pm \mathrm{SD})$ & $11.7 \pm 3.3$ \\
\hline Genetics & $n / N(\%)$ \\
\hline rs1058587 CC & $212 / 310(68.4)$ \\
\hline rs1058587 CG & $88 / 310(28.4)$ \\
\hline rs1058587 GG & $10 / 310(3.22)$ \\
\hline Allele C & $513 / 620(82.7)$ \\
\hline Allele G & $109 / 620(17.5)$ \\
\hline Sociodemographic & $n / N(\%)$ \\
\hline Female & $271 / 310(87.4)$ \\
\hline Civil status: married & $174 / 282(61.7)$ \\
\hline Occupation: household duties & $109 / 287(38)$ \\
\hline Socioeconomic status: medium & $195 / 278(70.1)$ \\
\hline High educational level & $169 / 212(79.7)$ \\
\hline Current smoking & $49 / 272(18)$ \\
\hline RA-related & $n / N(\%)$ \\
\hline Polyautoimmunity: SS & $16 / 310(5.1)$ \\
\hline Familial autoimmunity & $47 / 272(17.3)$ \\
\hline $\mathrm{EAM}^{\&}$ & $117 / 303(38.6)$ \\
\hline Comorbidity $^{\#}$ & $225 / 305(73.7)$ \\
\hline Rheumatoid factor $(+)$ & $162 / 237(68.3)$ \\
\hline $\mathrm{ACPA}(+)$ & $107 / 133(80.4)$ \\
\hline ANA $(+)$ & $31 / 75(41.3)$ \\
\hline Erosive $(+)$ & $99 / 110(90)$ \\
\hline Methotrexate & $248 / 273(90.8)$ \\
\hline DMARDs, ever ${ }^{¥}$ & $83 / 117(71)$ \\
\hline Antimalarials, ever ${ }^{\mathfrak{E}}$ & $158 / 256(61.7)$ \\
\hline Steroids, ever ${ }^{æ}$ & $250 / 269(92.9)$ \\
\hline Biological therapy, ever $€$ & $37 / 256(14.4)$ \\
\hline Cardiovascular & $n / N(\%)$ \\
\hline Cardiovascular disease $^{\mathrm{A}}$ & $130 / 187(69.5)$ \\
\hline cIMT abnormal & 66/118 (55.9) \\
\hline Familial cardiovascular disease & $19 / 245(7.7)$ \\
\hline BMI: obesity & $18 / 118(15.2)$ \\
\hline BMI: overweight & $42 / 118(35.6)$ \\
\hline Physical activity & $20 / 121(16.5)$ \\
\hline Abnormal waist-hip ratio & $90 / 117(76.9)$ \\
\hline
\end{tabular}

ACPA: anti-citrullinated protein antibodies; ANA: anti-nuclear antibodies; BMI: body mass index; cIMT: carotid intima-media thickness; DMARDs: disease modifying antirheumatic drugs; EAMs: extra-articular manifestations; HLA: human leukocyte antigen; m: months; MICl: macrophage inhibitory cytokine-1; RA: rheumatoid arthritis; SD: standard deviation; SS: Sjögren's syndrome; y: years.

${ }^{\&} \mathrm{EAM}$ is defined as the presence of at least one of the following: nodules, skin ulcerations, episcleritis, scleritis, vasculitis, neuropathy, pleural effusion, pulmonary nodules, or pulmonary hypertension.

${ }^{\#}$ It is defined as the presence of at least one of the following: type 2 diabetes mellitus, dyslipidemia, kidney disease, anemia, osteoporosis, depression, fibromyalgia, acid peptic disease, epilepsy, or infections (e.g., hepatitises A, B, and C, tuberculosis, or malaria).

${ }^{¥}$ If patient had used at least one of the following: sulfasalazine, D-penicillamine, gold salts, and leflunomide.

${ }^{E}$ If patient had used at least one of the following: chloroquine and hydroxychloroquine.

${ }^{\mathfrak{x}}$ If patient had used at least one of the following: prednisolone, methylprednisolone, and deflazacort.

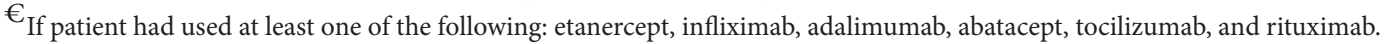

${ }^{A}$ It was categorized as positive if any of the following variables were present: hypertension, coronary artery disease, occlusive arterial disease, carotid disease, or thrombosis. 


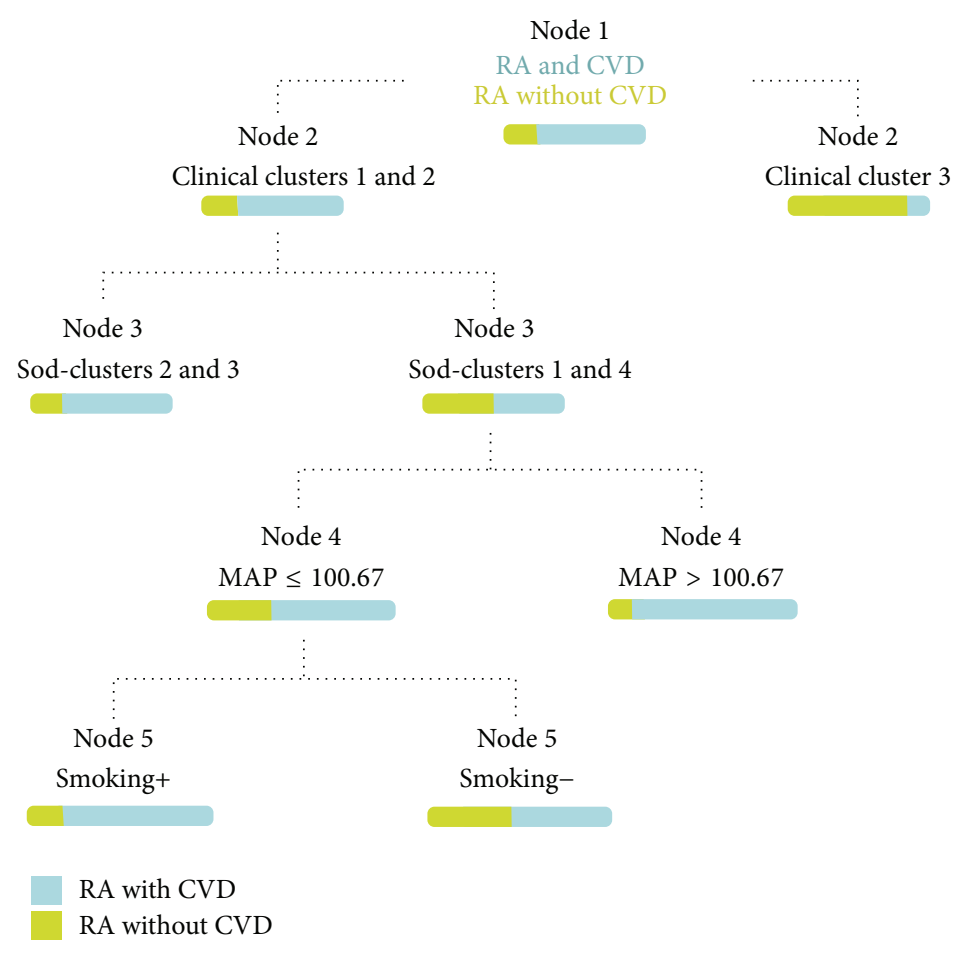

FIGURE 1: CART predictive model: the main predictive factors significantly associated with the development of CVD in RA are clinical and sociodemographic cluster, mean arterial pressure (MAP), and current smoking, with variable importance scores higher than 0.73 .

TABLE 2: GDF15 polymorphism (rs1058587) in Colombian patients with RA.

\begin{tabular}{lcc}
\hline Genotype & RA & CTR $^{*}$ \\
& $N=310(\%)$ & $N=228(\%)$ \\
\hline CC & $212(68.4)$ & $151(66.2)$ \\
CG & $88(28.4)$ & $70(30.7)$ \\
GG & $10(3.2)$ & $7(3.1)$ \\
Allele & $2 N=620$ & $2 N=456$ \\
C & $512(82.6)$ & $372(81.6)$ \\
G & $108(17.4)$ & $84(18.4)$ \\
\hline
\end{tabular}

* Significant differences were not observed.

(subphenotypes) in RA patients (Table 3) or with IMT were not found.

In Tables 4 and 5 are mentioned the variables associated with the presence of allele $\mathrm{C}$ and allele $\mathrm{G}$ of the $\mathrm{MIC1}$ rs1058587 SNP, respectively. Once again, we did not find associations between this SNP and CVD in those patients.

In the genotype analysis, the homozygotes $\mathrm{G} / \mathrm{G}$ were significantly older (median 54, IQR 11.7 years), had later AOD (median 43.7, IQR 9.92 years), and had the highest diastolic blood pressure (median 88.33, IQR $8.66 \mathrm{mmHg}$ ). On the other hand, the genotype $\mathrm{CC}$ was found as protective factor to have positive ANA (OR 0.27; 95\% C.I. 0.11-0.85, $P=0.019$ ).

3.1. CART Predictive Model. The CART model displayed a high proportion of patients with RA through clinical clusters 1 and 2 who also develop CVD (nodes 1 and 2). However, there was association between CVD and sod-cluster, mean arterial pressure (MAP), and current smoking; in fact the major association with CVD is in sod-clusters 2 and 3. However, sod-clusters 1 and 4 show a better discrimination for CVD (node 3). Moreover, in the same group of patients, CVD was higher in those with high MAP (i.e., more than $100 \mathrm{mmHg}$ ) (node 4). Finally, a greater proportion of CVD was observed in patients in node 4 with low MAP but presenting with current tobacco exposure (node 5) (Figure 1). This model had an AUC of 0.73 (cross-validated AUC), which represents an adequate predictive performance.

\section{Discussion}

Several previous studies of gene polymorphisms in patients with RA who had CVD had demonstrated genetic factors implicated in the development of this comorbidity, such as HLA-DRB1 shared epitope alleles [37, 38]. Moreover, the choice of genes for analysis in those studies was based on a relationship with RA per se or with inflammation. Herein, we were unable to replicate the influence of GDF15(MIC1) rs1058587 SNP in Colombian patients with RA and CVD as was previously observed in Swedish and Australian patients $[11,14]$. Differences in admixture patterns and allele frequencies among populations may account for the observed differences in the influence of genetic factors on disease. Our population is highly admixed with Amerindian, African, and Caucasian ancestries as compared with Australians and Swedish who are mainly Caucasians. The rs $1058587 \mathrm{G}$ allele 
TABLE 3: Characteristics associated with CVD in RA patients.

\begin{tabular}{lccc}
\hline & CVD & Non-CVD & \\
Variable & $130 / 187(69.5)$ & $57 / 187(30.5)$ & $P$ value \\
& Median (IQR) & Median (IQR) & $<0.001$ \\
\hline Duration of the disease (y) & $14.6(9.038)$ & $9.962(9.559)$ & 0.028 \\
EAM (m) & $17.115(37.6)$ & $95.491(11.605)$ & 0.0361 \\
Mean arterial pressure & $99.709(12.992)$ & Non-ECV & OR (95\% CI) \\
\hline Variable & ECV & $n / N(\%)$ & $33.93(8.6-259.7)$ \\
\hline Clinical cluster 1 ${ }^{\&}$ & $n / N(\%)$ & $32 / 57(56.1)$ & $1.99(1.07-4.16)$ \\
Time cluster 1 & $112 / 130(86.1)$ & $24 / 57(42.1)$ & $2.54(1.01-8.45)$ \\
Current smoking & $62 / 130(47.6)$ & $4 / 57(7.4)$ & - \\
GDF15 rs1058587 CC & $29 / 130(22.3)$ & $36 / 57(63.1)$ & - \\
GDF15 rs1058587 CG & $89 / 130(68.4)$ & $21 / 57(36.8)$ & - \\
GDF15 rs1058587 GG & $35 / 130(26.9)$ & $0 / 57(0)$ & - \\
Allele C & $6 / 130(4.6)$ & $93 / 114(81.5)$ & NS \\
Allele G & $213 / 260(81.9)$ & $21 / 114(18.4)$ & NS \\
\hline
\end{tabular}

CVD: cardiovascular disease; EAMs: extra-articular manifestations; GDF15: growth differentiation factor 15; IQR: interquartile range; y: years; m: months; NS: not significant; RA: rheumatoid arthritis.

${ }^{\&}$ Clinical cluster 1 corresponds to patients with the highest frequency of comorbidity, with moderate frequency of EAM, and without Sjögren’s syndrome.

${ }^{\#}$ Time cluster 1 corresponds to patients with later age at onset and shorter duration of the disease.

TABLE 4: Characteristics associated with allele C of the GDF15 rs1058587 SNP in RA patients.

\begin{tabular}{lccc}
\hline & Allele C (CC/CG) & Allele G (GG) & \\
Variable & $300 / 310(96.7)$ & $10 / 310(3.22)$ & $P$ value \\
& Median (IQR) & $88.333(8.66)$ & 0.0286 \\
\hline Diastolic blood pressure & $80.662(10.437)$ & $29.322(4.177)$ & 0.0457 \\
Body mass index & $25.326(4.691)$ & Allele G & OR (95\% CI) \\
\hline Variable & Allele C & $n / N(\%)$ & $0.14(0.02-0.80)$ \\
\hline Current smoking & $n / N(\%)$ & $4 / 10(40)$ & $0.09(0.009-0.97)$ \\
Obesity & $45 / 300(15)$ & $3 / 10(30)$ & 0.016 \\
CVD & $15 / 300(5)$ & $6 / 10(60)$ & 0.0175 \\
\hline
\end{tabular}

CVD: cardiovascular disease; IQR: interquartile range; RA: rheumatoid arthritis.

TABLE 5: Characteristics associated with allele G of the GDF15 rs1058587 SNP in RA patients.

\begin{tabular}{|c|c|c|c|c|}
\hline \multirow{3}{*}{ Variable } & Allele G (GG/CG) & Allele C (CC) & & \multirow{3}{*}{$P$ value } \\
\hline & $98 / 310(31.6)$ & $212 / 310(68.3)$ & & \\
\hline & Median (IQR) & Median (IQR) & & \\
\hline Age (y) & $49.398(12.46)$ & $53.986(11.976)$ & & 0.001 \\
\hline Age at onset (y) & 37.469 (11.513) & $41.344(12.59)$ & & 0.008 \\
\hline \multirow{2}{*}{ Variable } & Allele G & Allele C & \multirow{2}{*}{ OR (95\% CI) } & \multirow{2}{*}{$P$ value } \\
\hline & $n / N(\%)$ & $n / N(\%)$ & & \\
\hline Clinical cluster $1^{\&}$ & $72 / 98(73.46)$ & $129 / 212(60.84)$ & $2.88(1.16-9.32)$ & 0,0145 \\
\hline ANA $(+)$ & 15/98 (15.30) & $16 / 212(7.54)$ & $2.72(1.15-8.21)$ & 0,0203 \\
\hline Sjögren's syndrome & 4/98 (4.08) & $26 / 212(12.26)$ & $0.28(0.11-0.91)$ & 0,0209 \\
\hline Time cluster $1^{\#}$ & $32 / 98(32.63)$ & $98 / 212(46.22)$ & $0.52(0.31-0.92)$ & 0,0228 \\
\hline CVD & 41/98 (41.88) & $89 / 212(41.98)$ & & NS \\
\hline
\end{tabular}

ANA: anti-nuclear antibodies; CVD: cardiovascular disease; IQR: interquartile range; RA: rheumatoid arthritis.

${ }^{\&}$ Clinical cluster 1 corresponds to patients with the highest frequency of comorbidity, with moderate frequency of EAM, and without Sjögren's syndrome.

${ }^{\#}$ Time cluster 1 corresponds to patients with later age at onset and shorter duration of the disease. 
frequency was significantly lower in Colombians than in Australians and Swedish [11, 14].

The general prevalence of CVD in our cohort was 69.5\%. Some associations between RA patients with CVD were found. Variables that were significantly associated include smoking, higher MAP, longer duration of disease with EAM, clinical cluster number 1 , and time cluster number 1 . It is important to highlight that these associations encompass only clinical variables that include modifiable traditional risk factors for CVD like smoking and nontraditional risk factors including duration of disease and EAM [5].

GDF15(MIC1) rs1058587 C allele was nevertheless associated with minor frequency of smoking and obesity, lower $\mathrm{BMI}$, and lower measurements of diastolic blood pressure (Table 4), whereas G allele was associated with earlier AOD, clinical cluster number 1 , and positive ANA and it was inversely associated with SS and time cluster number 1. Although modest, these findings contrast with reports showing a specific interaction between the $\mathrm{G}$ allele and smoking, with an increase of risk for stroke [11].

At the genotype level a statistically significant association was found between $\mathrm{G} / \mathrm{G}$ genotype and high diastolic blood pressure, while $\mathrm{C} / \mathrm{C}$ genotype was inversely associated with positive ANA, suggesting a protective role for polyautoimmunity. We were not able to find other studies reporting associations between the rs1058587 homozygote forms and CVD or RA per se. As it is shown, these associations do not confirm a direct relationship between $M I C 1$ and CVD.

Many reasons exist why no significant association was elicited within the cohort and the gen evaluated with the presence of CVD. One of them is the sample size calculation since it was a nonprobability sample and all RA patients from this region were not included in the study. Besides, the heterogeneity of the cohort may prevent us from finding a direct association. A group of patients studied constitute a closed subpopulation into the admixed Colombian community (i.e., Medellín) and another group represents a major variation in ancestry. Otherwise, previous publications addressing the influence of GDF15(MIC1) in CVD were made in general population, not in RA patients. Therefore, the interaction among autoimmunity, admixture population, and genetic, epigenetic, and environmental factors could influence the behavior of GDF15(MIC1) in our study.

\section{Limitations of the Study}

The aim of this study was to determine the influence of rs1058587 SNP within GDF15(MIC1) gene on the risk of CVD in a Colombian RA population and we are aware of our study limitations. First of all, selection bias could be present in our analysis as not all patients from this region with RA were systematically included. Secondly, although the study sample size is not negligible, it would have been more valuable to have had an appropriate follow-up to establish valid associations between CVD, GDF15(MIC1), and RA. This, in turn, could have improved both internal and external validities. Finally, the cross-sectional nature of the study does not allow us to infer causality. Although we were able to evaluate some associations between GDF15(MIC1) and risk factors for CVD, it would have been more valuable to have a follow-up, as it is possible in cohort multicenter studies, to validate and establish more associations between this gene, CVD, and RA.

\section{Conclusions}

Contrary to prior reports, we could not find an association between the SNP rs1058587 of the gene GDF15 and the development of CVD (measured through subphenotypes or IMT) in the population studied. There was an association between this SNP and traditional and environmental factors for CVD (i.e., age, obesity, and smoking) and some nontraditional risk factors (i.e., ANA and SS). However, we had not found direct association between the polymorphism and CVD. In fact, MAP, EAM, smoking, duration of the disease, and AOD are highlighting the pivotal role of these factors in our population. CVD in RA patients should be approached following the $5 \mathrm{P}$ rule: (1) predicting patients at risk of developing CVD; (2) preventing CVD in susceptible patients; (3) personalizing the approach of CVD based on the premise that susceptibility and severity of the disease are unique in each patient; (4) allowing the patient to participate in the decisions made in order to prevent and treat CVD; and (5) incorporating all data and policies according to the population, since the characteristics and natural history of diseases are population-specific [39].

\section{Conflict of Interests}

The authors declare that there is no conflict of interests regarding the publication of this paper.

\section{Acknowledgments}

The authors are grateful to the members of the Center for Autoimmune Diseases Research (CREA) for fruitful discussions and to Parvathi Viswanathan for technical support. This work was supported by the Universidad del Rosario, Bogotá, Colombia.

\section{References}

[1] R. Sanmartí, V. Ruiz-Esquide, and M. V. Hernández, "Rheumatoid arthritis: a clinical overview of new diagnostic and treatment approaches," Current Topics in Medicinal Chemistry, vol. 13, no. 6, pp. 698-704, 2013.

[2] D. L. Scott, F. Wolfe, and T. W. J. Huizinga, "Rheumatoid arthritis," The Lancet, vol. 376, no. 9746, pp. 1094-1108, 2010.

[3] C. Barragán-Martínez, J. Amaya-Amaya, R. Pineda-Tamayo et al., "Gender differences in latin-american patients with rheumatoid arthritis," Gender Medicine, vol. 9, no. 6, pp. 490.e5510.e5, 2012.

[4] A. Chopra, "The WHO ILAR COPCORD Latin America: consistent with the world and setting a new perspective," Journal of Clinical Rheumatology, vol. 18, no. 4, pp. 167-169, 2012.

[5] O.-D. Ortega-Hernandez, R. Pineda-Tamayo, A. L. Pardo, A. Rojas-Villarraga, and J.-M. Anaya, "Cardiovascular disease is associated with extra-articular manifestations in patients with 
rheumatoid arthritis," Clinical Rheumatology, vol. 28, no. 7, pp. 767-775, 2009.

[6] A. Rojas-Villarraga, O.-D. Ortega-Hernandez, L. F. Gomez et al., "Risk factors associated with different stages of atherosclerosis in Colombian patients with rheumatoid arthritis," Seminars in Arthritis \& Rheumatism, vol. 38, no. 2, pp. 71-82, 2008.

[7] J. Amaya-Amaya, J. C. Sarmiento-Monroy, R.-D. Mantilla, R. Pineda-Tamayo, A. Rojas-Villarraga, and J.-M. Anaya, "Novel risk factors for cardiovascular disease in rheumatoid arthritis," Immunologic Research, vol. 56, no. 2-3, pp. 267-286, 2013.

[8] J. C. Sarmiento-Monroy, J. Amaya-Amaya, J. S. EspinosaSerna, C. Herrera-Díaz, J.-M. Anaya, and A. Rojas-Villarraga, "Cardiovascular disease in rheumatoid arthritis: a systematic literature review in Latin America," Arthritis, vol. 2012, Article ID 371909, 17 pages, 2012.

[9] A. Sandoo, J. J. C. S. V. van Zanten, G. S. Metsios, D. Carroll, and G. D. Kitas, "Vascular function and morphology in rheumatoid arthritis: a systematic review," Rheumatology (Oxford), vol. 50, no. 11, pp. 2125-2139, 2011.

[10] E. Gkaliagkousi, E. Gavriilaki, M. Doumas, K. Petidis, S. Aslanidis, and D. Stella, "Cardiovascular risk in rheumatoid arthritis: pathogenesis, diagnosis, and management," Journal of Clinical Rheumatology, vol. 18, no. 8, pp. 422-430, 2012.

[11] L. Ärlestig and S. Rantapää-Dahlqvist, "Polymorphisms of the genes encoding CD40 and growth differentiation factor 15 and in the 9p21.3 region in patients with rheumatoid arthritis and cardiovascular disease," Journal of Rheumatology, vol. 39, no. 5, pp. 939-945, 2012.

[12] K. Unsicker, B. Spittau, and K. Krieglstein, “The multiple facets of the TGF- $\beta$ family cytokine growth/differentiation factor15/macrophage inhibitory cytokine-1," Cytokine \& Growth Factor Reviews, vol. 24, no. 4, pp. 373-384, 2013.

[13] R. S. Mehta, M. Song, N. Bezawada et al., "A Prospective study of macrophage inhibitory cytokine-1 (MIC-1/GDF15) and risk of colorectal cancer," Journal of the National Cancer Institute, vol. 106, no. 4, Article ID dju016, 2014.

[14] D. A. Brown, J. Moore, H. Johnen et al., "Serum macrophage inhibitory cytokine 1 in rheumatoid arthritis: a potential marker of erosive joint destruction," Arthritis \& Rheumatism, vol. 56, no. 3, pp. 753-764, 2007.

[15] F. C. Arnett, S. M. Edworthy, D. A. Bloch et al., "The American Rheumatism Association 1987 revised criteria for the classification of rheumatoid arthritis," Arthritis and Rheumatism, vol. 31, no. 3, pp. 315-324, 1988.

[16] Law 115, General on Education, 8th February 1994.

[17] Law 30, General on Higher Education, p. 4, 1992.

[18] J. Cárdenas-Roldán, A. Rojas-Villarraga, and J.-M. Anaya, "How do autoimmune diseases cluster in families? A systematic review and meta-analysis," BMC Medicine, vol. 11, no. 1, article 73, 2013.

[19] D. van der Heijde, "How to read radiographs according to the Sharp/van der Heijde method," The Journal of Rheumatology, vol. 26, no. 3, pp. 743-745, 1999.

[20] British Cardiac Society, British Hyperlipidaemia Association, British Hypertension Society, and British Diabetic Association, "Joint British recommendations on prevention of coronary heart disease in clinical practice: summary," British Medical Journal, vol. 320, pp. 705-708, 2000.

[21] P. G. Talwalkar, C. G. Sreenivas, A. Gulati, and H. Baxi, "Journey in guidelines for lipid management: from adult treatment panel (ATP)-I to ATP-III and what to expect in ATP-IV,' Indian
Journal of Endocrinology and Metabolism, vol. 17, pp. 628-635, 2013.

[22] G. Veronesi, F. Gianfagna, S. Giampaoli et al., "Improving longterm prediction of first cardiovascular event: the contribution of family history of coronary heart disease and social status," Preventive Medicine, vol. 64, pp. 75-80, 2014.

[23] B. A. Franklin, J. L. Durstine, C. K. Roberts, and R. J. Barnard, "Impact of diet and exercise on lipid management in the modern era," Best Practice \& Research: Clinical Endocrinology \& Metabolism, vol. 28, no. 3, pp. 405-421, 2014.

[24] American Diabetes Association, "Standards of medical care in diabetes-2012," Diabetes Care, vol. 35, supplement 1, pp. S11S63, 2012.

[25] Z. Reiner, A. L. Catapano, G. de Backer et al., "ESC/EAS guidelines for the management of dyslipidaemias: the task force for the management of dyslipidaemias of the European Society of Cardiology (ESC) and the European Atherosclerosis Society (EAS)," European Heart Journal, vol. 32, no. 14, pp. 1769-1818, 2011.

[26] D. W. Jones and J. E. Hall, "Seventh report of the Joint National Committee on Prevention, Detection, Evaluation, and Treatment of High Blood Pressure and evidence from new hypertension trials," Hypertension, vol. 43, no. 1, pp. 1-3, 2004.

[27] Y. Liao, S. Kwon, S. Shaughnessy et al., "Critical evaluation of adult treatment panel III criteria in identifying insulin resistance with dyslipidemia," Diabetes Care, vol. 27, no. 4, pp. 978983, 2004.

[28] S. Klein, D. B. Allison, S. B. Heymsfield et al., "Waist circumference and cardiometabolic risk: a consensus statement from shaping America's health: Association for weight management and obesity prevention; NAASO, the obesity society; the American society for nutrition; and the American diabetes association," Obesity, vol. 15, no. 5, pp. 1061-1067, 2007.

[29] C. Day, "Metabolic syndrome, or what you will: definitions and epidemiology," Diabetes \& Vascular Disease Research, vol. 4, no. 1, pp. 32-38, 2007.

[30] P. C. Deedwania, K. C. Maki, M. R. Dicklin, N. J. Stone, C. M. Ballantyne, and M. H. Davidson, "Application of recent definitions of the metabolic syndrome to survey data from the National Cholesterol Education Program Evaluation Project Utilizing Novel E-Technology (NEPTUNE II)," Journal of the CardioMetabolic Syndrome, vol. 1, no. 5, pp. 295-300, 2006.

[31] I. Wendelhag, O. Wiklund, and J. Wikstrand, "Arterial wall thickness in familial hypercholesterolemia: ultrasound measurement of intima-media thickness in the common carotid artery," Arteriosclerosis and Thrombosis, vol. 12, no. 1, pp. 70-77, 1992.

[32] R. Salonen and J. T. Salonen, "Determinants of carotid intimamedia thickness: a population-based ultrasonography study in Eastern Finnish men," Journal of Internal Medicine, vol. 229, no. 3, pp. 225-231, 1991.

[33] P. Pignoli, E. Tremoli, A. Poli, P. Oreste, and R. Paoletti, "Intimal plus medial thickness of the arterial wall: a direct measurement with ultrasound imaging," Circulation, vol. 74, no. 6, pp. 1399$1406,1986$.

[34] G. Mancia, G. de Backer, A. Dominiczak et al., "2007 guidelines for the management of arterial hypertension: the Task Force for the Management of Arterial Hypertension of the European Society of Hypertension (ESH) and of the European Society of Cardiology (ESC)," European Heart Journal, vol. 28, pp. 14621536, 2007. 
[35] L. Morineau, A. Lebart, and M. Piron, Statistique Exploratoire Multidimensionnelle, Dunod, Paris, France, 1995.

[36] R Development Core Team, R: A Language and Environment for Statistical Computing, R Foundation for Statistical Computing, Vienna, Austria, 2013.

[37] M. A. Gonzalez-Gay, C. Garcia-Porrua, and A. H. Hajeer, "Influence of human leukocyte antigen-DRB1 on the susceptibility and severity of rheumatoid arthritis," Seminars in Arthritis and Rheumatism, vol. 31, no. 6, pp. 355-360, 2002.

[38] E. W. Karlson, L. B. Chibnik, J. Cui et al., "Associations between Human leukocyte antigen, PTPN22, CTLA4 genotypes and rheumatoid arthritis phenotypes of autoantibody status, age at diagnosis and erosions in a large cohort study," Annals of the Rheumatic Diseases, vol. 67, no. 3, pp. 358-363, 2008.

[39] C. Auffray, D. Charron, and L. Hood, "Predictive, preventive, personalized and participatory medicine: back to the future," Genome Medicine, vol. 2, no. 8, article 57, 2010. 


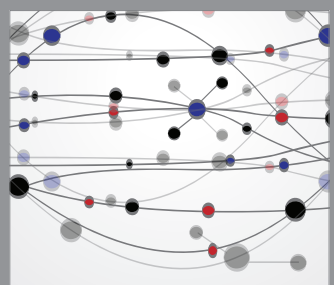

The Scientific World Journal
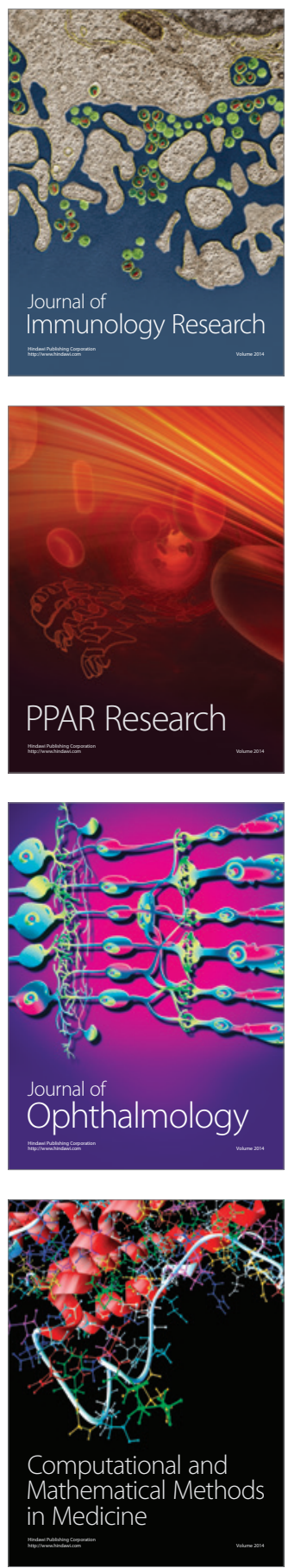

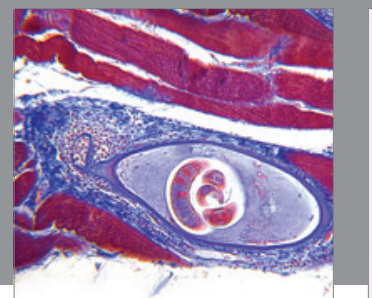

Gastroenterology

Research and Practice
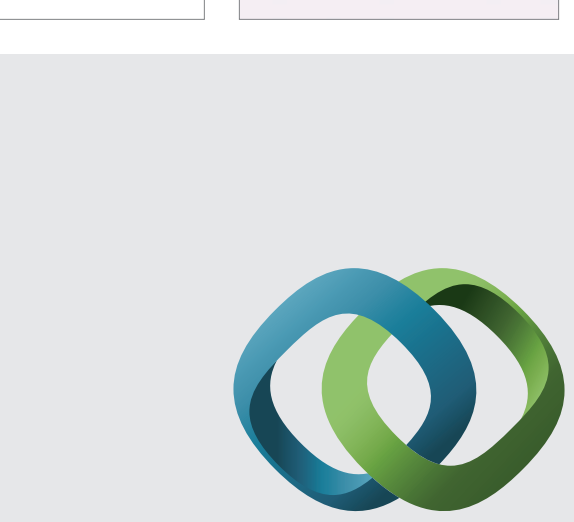

\section{Hindawi}

Submit your manuscripts at

http://www.hindawi.com
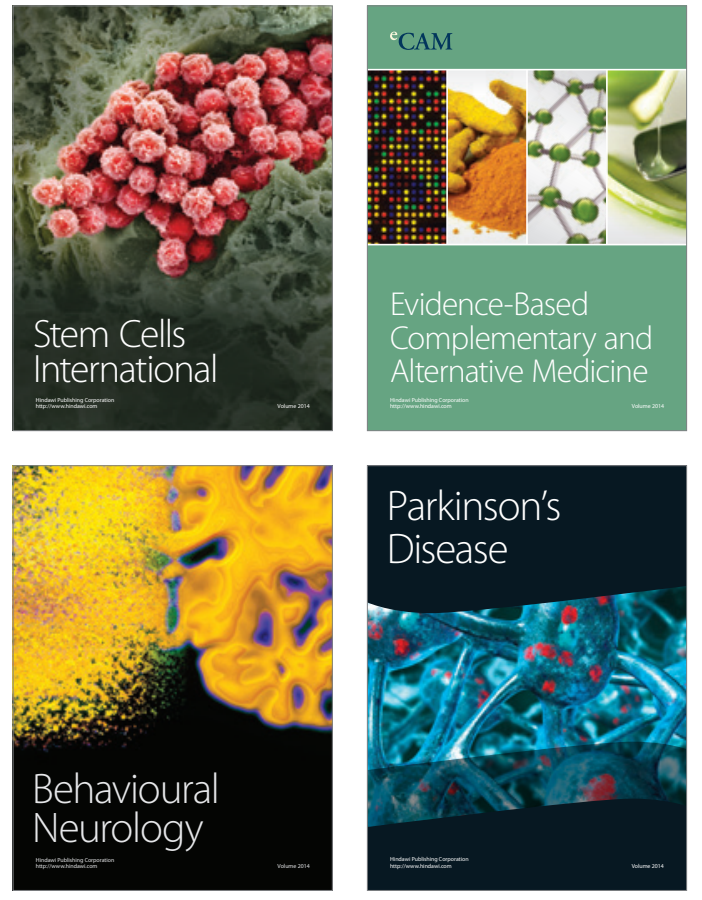
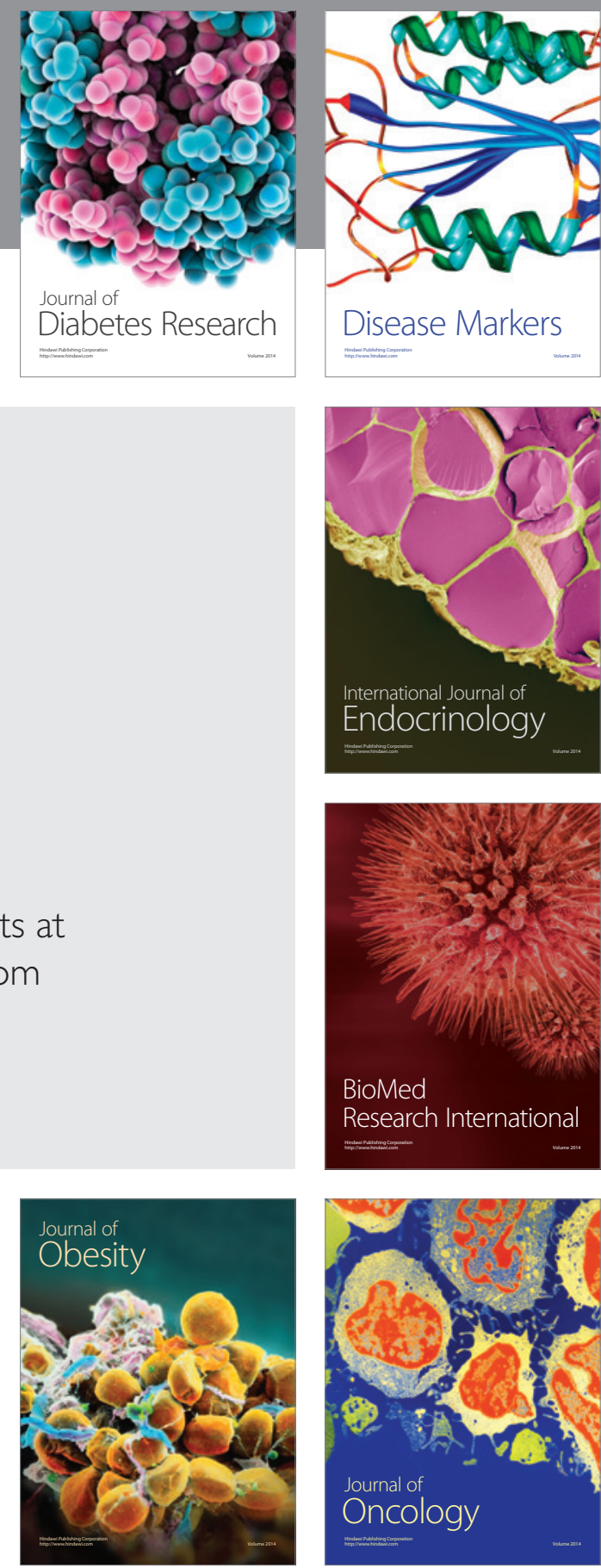

Disease Markers
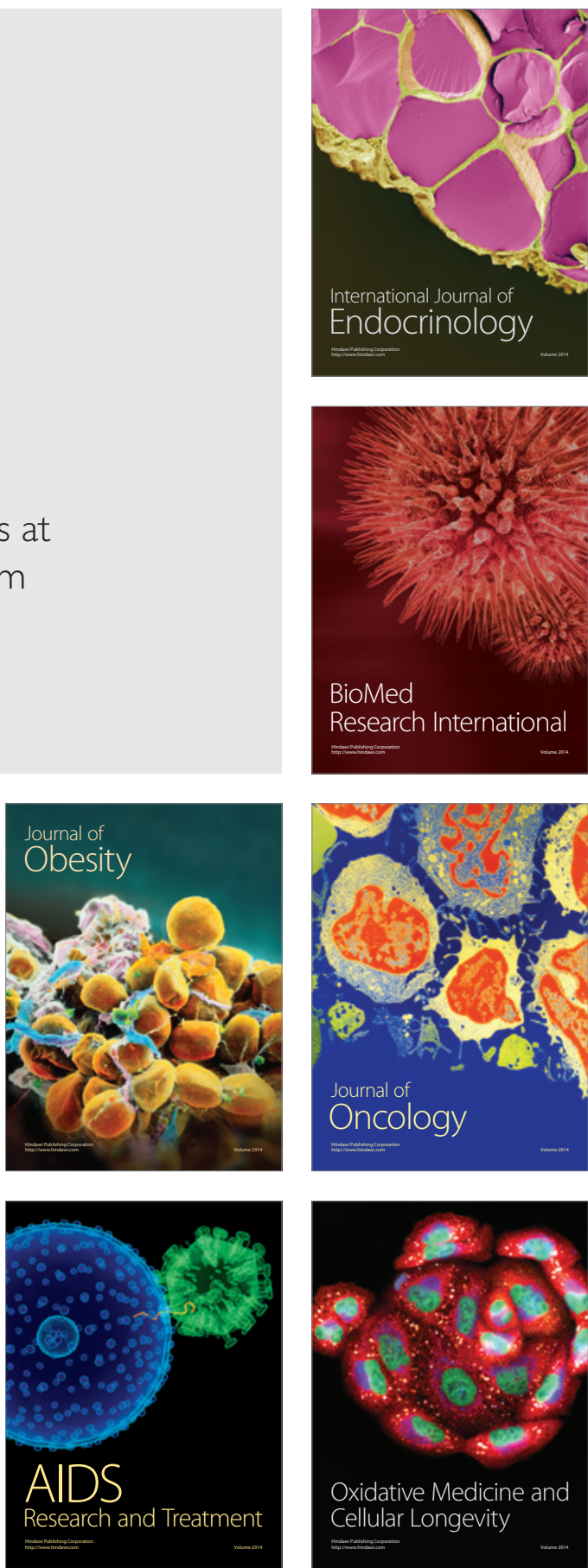